\title{
Achieving accurate FTIR measurements on high performance bandpass filters
}

Book or Report Section

Accepted Version

Hunneman, R., Sherwood, R., Deeley, C. and Spragg, R. (1998) Achieving accurate FTIR measurements on high performance bandpass filters. In: deHaseth, J.A. (ed.) Fourier Transform Spectroscopy. AIP Conference Proceedings (430). American Institute of Physics, pp. 435-438. ISBN 1563967464 Available at http://centaur.reading.ac.uk/978/

It is advisable to refer to the publisher's version if you intend to cite from the work. See Guidance on citing.

Published version at: http://proceedings.aip.org/proceedings/confproceed/430.jsp

Publisher: American Institute of Physics

Publisher statement: Copyright (1998) American Institute of Physics. This article may be downloaded for personal use only. Any other use requires prior permission of the author and the American Institute of Physics

All outputs in CentAUR are protected by Intellectual Property Rights law, including copyright law. Copyright and IPR is retained by the creators or other copyright holders. Terms and conditions for use of this material are defined in 
the End User Agreement.

www.reading.ac.uk/centaur

\section{CentAUR}

Central Archive at the University of Reading

Reading's research outputs online 


\title{
Achieving Accurate FTIR Measurements on High Performance Bandpass Filters
}

\author{
R Hunneman ${ }^{1}$, R Sherwood $^{1}, \mathrm{C}$ Deeley $^{2}$ and $\underline{\mathrm{R}}$ Spragg $^{2}$ \\ 1. University of Reading, Infrared Multilayer Laboratory, Department of Cybernetics, Whiteknights, Reading, Berks RG6 $2 A Y$ \\ England, 2.Perkin-Elmer Ltd. Post Office Lane, Beaconsfield, Bucks HP9 1QA England
}

The sources of ordinate error in FTIR spectrometers are reviewed with reference to measuring small out-of-band features in the spectra of bandpass filters. Procedures for identifying instrumental artefacts are described. It is shown that features well below $0.01 \% \mathrm{~T}$ can be measured reliably.

\section{INTRODUCTION}

The optical industry has been slow to adopt Fourier Transform instrumentation for critical transmission measurements because of concerns about their ordinate accuracy. In this paper we look at the problems in characterizing a set of bandpass filters required for a satellite-borne instrument. Particular emphasis is placed on the measurement of weak features in the blocking regions of bandpass filters. The procedures we have adopted to identify any residual effects due to double modulation or non-linearities are reported. Out-of-band features can be reliably measured at levels well below $0.01 \% \mathrm{~T}$.

\section{PROBLEMS IN FT INSTRUMENTS}

There are two main types of potential problem affecting these measurements. One is that of interreflections between surfaces in the optical train. Such problems are common to all optical spectrometers but their consequences in interferometers are different from those in dispersive instruments. The second is that of non-linearities in the detector and subsequent electronics. These problems are accentuated in FT instruments because of the high dynamic range resulting from the multiplex advantage. The consequences of the problems are seen both in inaccurate transmittance values and in the presence of spectral artefacts. The artefacts typically appear at multiples of the true wavenumber and so represent a significant problem in measuring the blocking region of bandpass filters.

In the example of Fig. 1 the filter passband starts at about $1500 \mathrm{~cm}-$ 1. There is apparent structure in the blocking region below $3000 \mathrm{~cm}-1$, Fig. 2, but most of this is due to the spectrometer rather than the sample. This can be demonstrated by measuring the spectrum in the presence of a sapphire window that is opaque below $1600 \mathrm{~cm}-1$.

\section{Interreflections}

Surfaces in the optical train are angled where practicable to deflect unwanted reflections. When interreflections involve the interferometer double modulation can occur, leading to artefacts outside the passband of the filter. The main problem is the reflection between the sample and interferometer. Masking can be used to intercept the reflections when tilting the sample is not acceptable. Doing this reduces the artefacts by about $80 \%$, to below $0.2 \% \mathrm{~T}$, Fig. 3 . There are also reflections on the source side that can be removed by further masking. The resultant overall reduction in throughput of the spectrometer is little more than $50 \%$. The residual features seen in Fig. 3 around 2400 to $2300 \mathrm{~cm}-1$ are genuine, also being observed when the passband is blocked using sapphire.

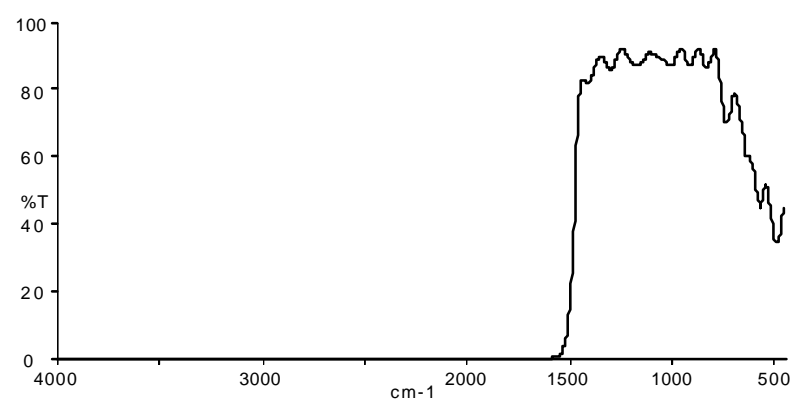

FIGURE 1. Spectrum of typical longpass filter.

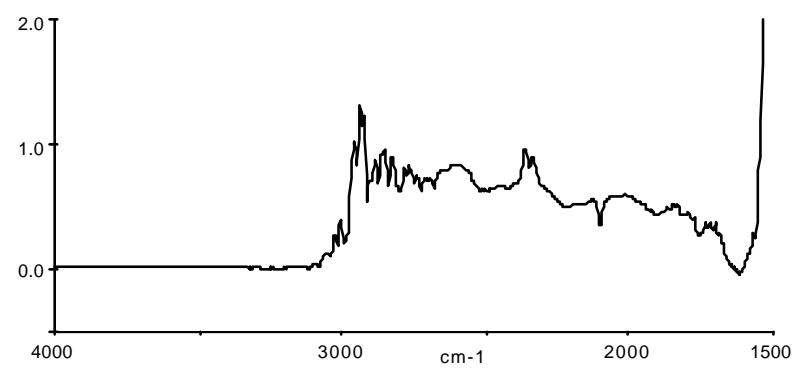

FIGURE 2. Spurious features observed in the blocking region.

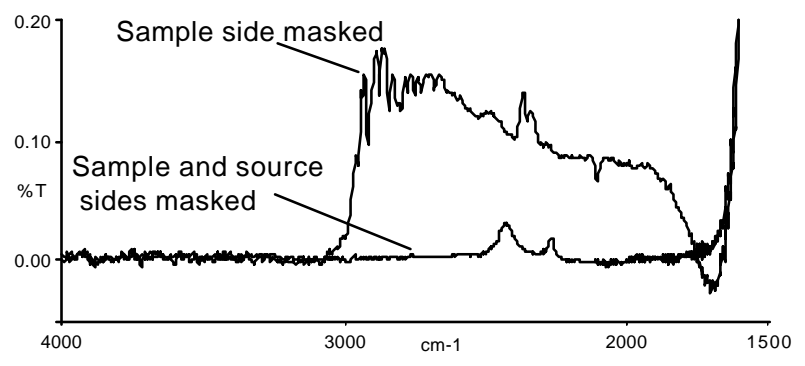

FIGURE 3. Spectra obtained with masking to block any interreflections involving the interferometer. 


\section{Residual Effects}

With reflections eliminated lower level artefacts become apparent. The first of these is ripple caused by apodization. The commonly used Norton-Beer functions were originally identified as providing the best compromise between the amplitude of the first sidelobe and band broadening. However as the functions do not go to zero at the maximum OPD the sidelobe intensities decay rather slowly. In Fig. 3 the sidelobes resulting from stong Norton-Beer apodization are clearly visible around $2000 \mathrm{~cm}-1$. For these measurments a function providing more rapid decay of sidelobe intensity, such as Filler, may be more appropriate.

Errors in the digitization of the interferogram can also introduce artefacts. If the number of bits used for digitization is inadequate the spectrum shows what looks like high frequency noise that is not reduced by signal averaging. The artefects caused by inaccuracies in the $\mathrm{A} / \mathrm{D}$ conversion typically have broader structure. Figure 4 . shows the effect of a single bit A/D conversion error on the spectrum of the filter in Fig. 1.

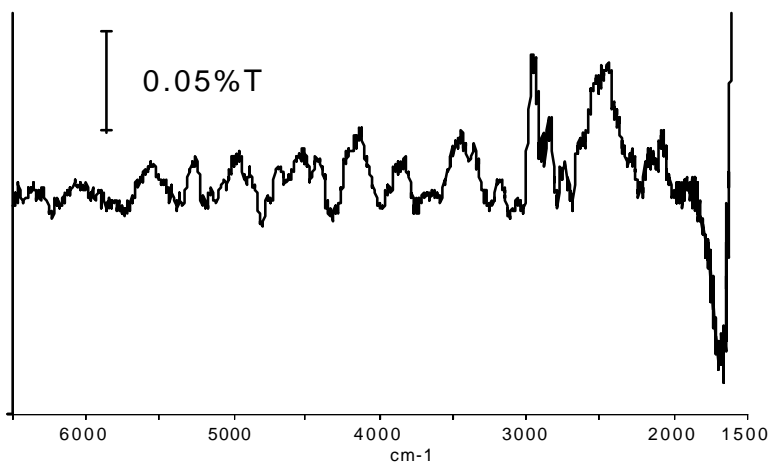

FIGURE 4. Out-of-band region measured with a single bit A/D conversion error.

\section{TESTING PERFORMANCE}

All the measurements are from Perkin-Elmer S2000 Optica instruments incorporating the features described earlier. Although very low noise levels are needed, DTGS detectors are used because of the extreme demands placed on linearity by these high dynamic range measurements. The overall ordinate accuracy is checked with samples of known refractive index, germanium and zinc selenide.

The spectrum from a high performance band pass filter is shown in Fig. 5. This was taken with 256 scans at $8 \mathrm{~cm}-1$ resolution in approximately 8 minutes. Transmission at $1596 \mathrm{~cm}-1$ is around $40 \%$. The small features around 3200 and $4800 \mathrm{~cm}-1$ vanish when the region below $2000 \mathrm{~cm}-1$ is blocked with a glass slide, showing that these features are not genuine. The origin of the feature at $3200 \mathrm{~cm}-1$ could be optical or electronic. However tilting the sample has little effect, showing that interreflections with the sample are not significant.

The amplitude of these artefacts varies with the level of analog gain used in processing the detector signal. It is minimized by using the maximum gain, suggesting that they result from limitations in the digital signal processing.
This example demonstrates that any residual artefacts appear at or below the level of $0.01 \% \mathrm{~T}$. These artefacts are found at multiples of the true wavenumbers. If it is possible to find a suitable material to block the passband measurements can be made at levels well below $0.01 \% \mathrm{~T}$.

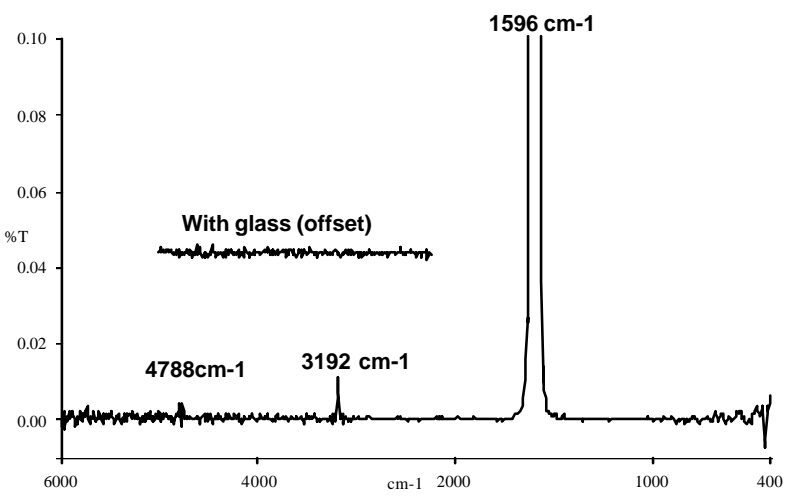

FIGURE 5. Spectrum of a high performance band pass filter showing the level of measurement artefacts.

\section{THE HIRDLS INSTRUMENT}

The High Resolution Dynamics Limb Sounder (HIRDLS) is a filter-based limb-viewing radiometer instrument scheduled to fly on the EOS CHEM satellite to be launched in 2002. The performance of the instrument is critically dependant on that of the filters used and the knowledge of their spectral characteristics. HIRDLS has 21 channels covering the spectral range from 6 to $17.5 \mu \mathrm{m}$, with multilayer interference filters using Lead Telluride, Germanium, Zinc Selenide and Zinc Sulphide as layer materials. The channels are defined by a set of filters operating at $300 \mathrm{~K}$ with bandwidths of between 1 and $8 \%$ (FWHM) with an additional set of bandpass filters operating at $65 \mathrm{~K}$ at the cold focal plane which provide further blocking and to reduce cross-channel / height spectral contamination. Thus there are 42 sets of filter measurements needed, encompassing a transmission range of $0.01 \% \mathrm{~T}$ to nearly $100 \%$.T, reflecting the required knowledge level of the filter blocking and that of the antireflection coating performance. Additionally in the case of the cold filters these measurements have to be made at $65 \mathrm{~K}$.

\section{Measuring out-of-band Features}

The filter used to illustrate low level measurements has a passband centered at about $580 \mathrm{~cm}-1$, Fig. 6. The out-of-band region of this filter has features with transmittance of up to $0.5 \% \mathrm{~T}$. It should be pointed out that this leakage is not critical in the HIRDLS design because other elements in the system will remove it. It is clear from measurements incorporating a $2.5 \mathrm{~mm}$ zinc sulfide window, that all the features seen at the $0.1 \%$ level in Fig. 7 are genuine. Closer examination in Fig. 8 shows artefacts around $1750-1600$ and $1200-1100 \mathrm{~cm}-1$ at the $0.01 \% \mathrm{~T}$ level. The artefact between 1200 and $1100 \mathrm{~cm}-1$ overlaps with a genuine feature but its presence is evident when the spectra are overlaid. Another way of 
identifying this and other small artefacts is that their appearance is very sensitive to the phase calculation for the spectrum.

The observed spectrum is also compared with a calculated spectrum obtained using the FilmStar program ${ }^{1}$ in Fig. 9. The agreement is extremely good at longer wavelengths. At shorter wavelengths the calculation is less satisfactory, with the calculated features displaced to shorter wavelengths than those observed. This is because the properties of lead telluride are less well modelled and the orders of interference are higher.

\section{SUMMARY}

These measurements demonstrate that spectral artefacts are typically smaller than $0.01 \% \mathrm{~T}$ even when the passband is not blocked.

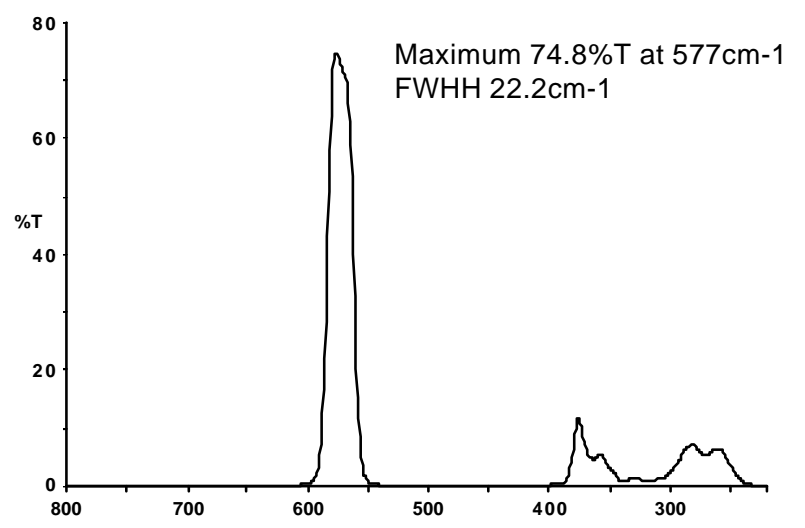

FIGURE 6. Passband of HIRDLS filter.

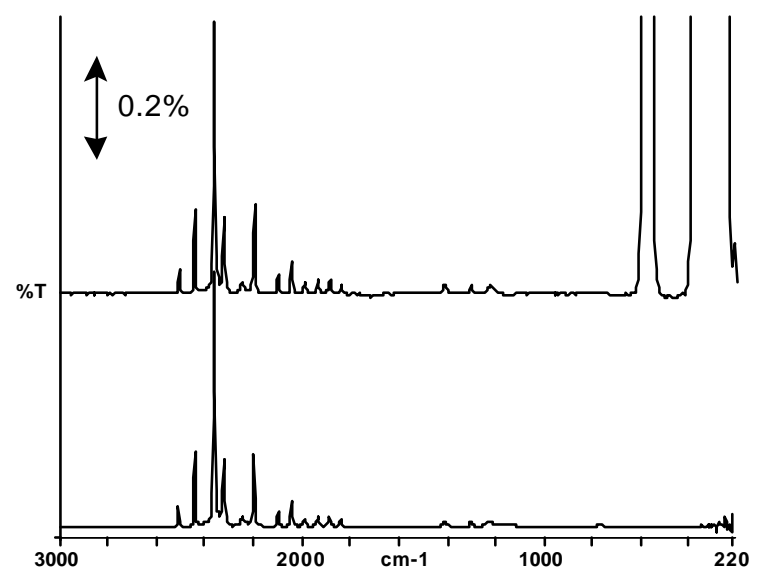

FIGURE 7. Out of band features of HIRDLS filter. Upper: full spectrum, lower: with $\mathrm{ZnS}$ blocking the passband.

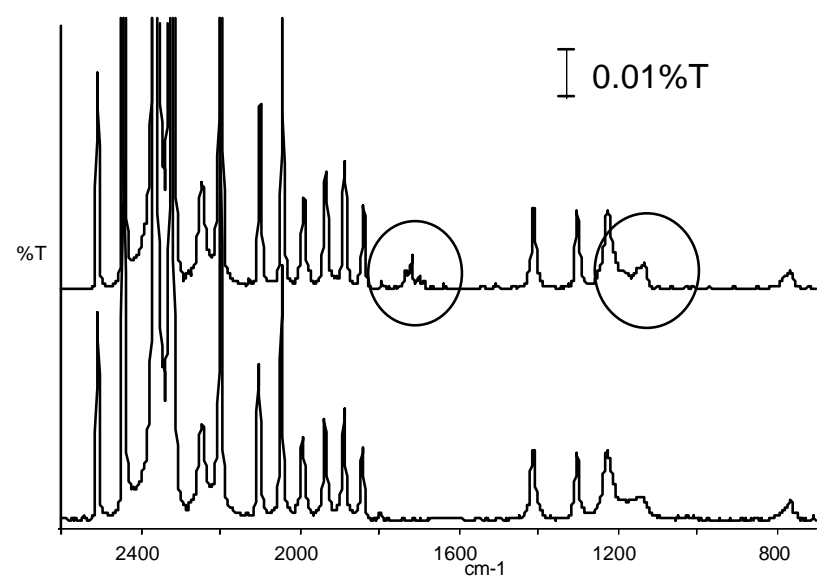

FIGURE 8. Exanded detail of the spectra of Fig.6. The upper spectrum shows artefacts near 1150 and $1750 \mathrm{~cm}-1$ that are absent in the lower spectrum with the passband blocked.

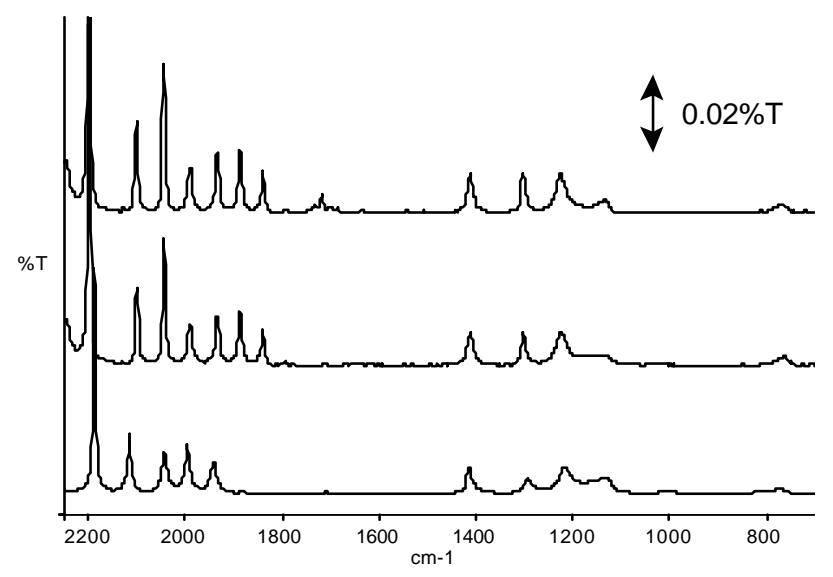

Figure 9. Comparison of observed and calculated out-of-band features. Top: full spectrum, center: observed with passband blocked, bottom: calculated.

\section{ACKNOWLEDGEMENT}

We are grateful to Dr Gary Hawkins of the Reading Infrared Multilayer Laboratory for the calculated spectrum of the HIRDLS filter.

\section{REFERENCE}

1. FTG Software Associates, Princeton NJ 08642 